Article

\title{
Regulation of $\mathrm{K}^{+}$Conductance by a Hydrogen Bond in Kv2.1, Kv2.2, and Kv1.2 Channels
}

\author{
Yuchen Zhang ${ }^{\dagger}$, Xuefeng Zhang ${ }^{\dagger}$, Cuiyun Liu and Changlong $\mathrm{Hu}$ *(D)
}

check for updates

Citation: Zhang, Y.; Zhang, X.; Liu,

C.; $\mathrm{Hu}, \mathrm{C}$. Regulation of $\mathrm{K}^{+}$

Conductance by a Hydrogen Bond in Kv2.1, Kv2.2, and Kv1.2 Channels.

Membranes 2021, 11, 190.

https://doi.org/10.3390/

membranes 11030190

Academic Editor: Tanima Bose

Received: 11 January 2021

Accepted: 5 March 2021

Published: 9 March 2021

Publisher's Note: MDPI stays neutral with regard to jurisdictional claims in published maps and institutional affiliations.

Copyright: (c) 2021 by the authors. Licensee MDPI, Basel, Switzerland. This article is an open access article distributed under the terms and conditions of the Creative Commons Attribution (CC BY) license (https:// creativecommons.org/licenses/by/ $4.0 /)$.
Department of Physiology and Biophysics, Institutes of Brain Science, School of Life Sciences, Fudan University, Shanghai 200032, China; 19110700020@fudan.edu.cn (Y.Z.); 18210700080@fudan.edu.cn (X.Z.); 15210700016@fudan.edu.cn (C.L.)

* Correspondence: clhu@fudan.edu.cn

+ The authors contributed equally to this work.

\begin{abstract}
The slow inactivation of voltage-gated potassium $(\mathrm{Kv})$ channels plays an important role in controlling cellular excitability. Recently, the two hydrogen bonds (H-bonds) formed by W434-D447 and T439-Y445 have been reported to control the slow inactivation in Shaker potassium channels. The four residues are highly conserved among $\mathrm{Kv}$ channels. Our objective was to find the roles of the two H-bonds in controlling the slow inactivation of mammalian Kv2.1, Kv2.2, and Kv1.2 channels by point mutation and patch-clamp recording studies. We found that mutations of the residues equivalent to W434 and T439 in Shaker did not change the slow inactivation of the Kv2.1, Kv2.2, and Kv1.2 channels. Surprisingly, breaking of the inter-subunit H-bond formed by W366 and Y376 (Kv2.1 numbering) by various mutations resulted in the complete loss of $\mathrm{K}^{+}$conductance of the three $\mathrm{Kv}$ channels. In conclusion, we found differences in the H-bonds controlling the slow inactivation of the mammalian $\mathrm{Kv}$ channels and Shaker channels. Our data provided the first evidence, to our knowledge, that the inter-subunit H-bond formed by W366 and Y376 plays an important role in regulating the $\mathrm{K}^{+}$conductance of mammalian Kv2.1, Kv2.2, and Kv1.2 channels.
\end{abstract}

Keywords: voltage-gated potassium channels; channel inactivation; hydrogen bonds; potassium conductance

\section{Introduction}

Mammalian voltage-gated potassium (Kv) channels, consisting of 12 subfamilies (Kv1-12), including 40 subtypes, play key roles in maintaining the membrane potential and mediating neuronal and muscular excitability and have been implicated in various neuronal and cardiovascular diseases [1-3]. Kv channels are tetramers forming a $\mathrm{K}^{+}$selective pore through four $\alpha$-subunits. Each $\alpha$-subunit comprises six transmembrane helices (S1-S6) and a re-entrant P loop between S5 and S6, which includes a pore helix and a selectivity filter. $\mathrm{Kv}$ channels commonly open at depolarization and enter an inactivated state under prolonged depolarization, which blocks $\mathrm{K}^{+}$conduction and plays a key role in controlling cellular excitability.

The inactivation of $\mathrm{Kv}$ channels includes two distinct processes, termed N-type (fast) and C-type (slow) inactivation [4]. Slow inactivation is presumably associated with the conformational changes in the selectivity filter region, which can be influenced by the pore-helix residues or the adjacent extracellular vestibule [5-8]. The mechanism underling the slow inactivation of $\mathrm{Kv}$ channels has been intensively studied on the bacterial KcsA and Drosophila shaker channels (in the following text, shaker stands for the Drosophila shaker channel.). Hydrogen bonds (H-bonds) between the selectivity filter and the adjacent pore helix are considered as major regulators of the slow inactivation. The two H-bonds formed by W67-D80 and E71-D80 have been shown to play key roles in controlling the slow inactivation of KcsA channels $[9,10]$. The W67 and D80 are highly conserved in Kv channels. Mutations at equivalent positions in Shaker channels (W434 and D447) lead 
to drastic changes in slow inactivation [11-14]. Using synthetic amino acids, Pless et al. recently demonstrated that an intra-subunit H-bond formed by W434-D447 and an intersubunit H-bond formed by T439-Y445 regulate the slow inactivation of Shaker channels [15]. Besides these four residues, T449 is also shown to play important roles in regulating the slow inactivation of Shaker channels [16]. However, mutations at this site do not result in similar effects on the slow inactivation of mammalian Kv1.4 and Kv1.5 channels [17,18], which suggests that the residues controlling slow inactivation for mammalian $\mathrm{Kv}$ channels and Shaker channels may be different. Compared to Shaker channels, the role of the $\mathrm{H}$ bonds in mammalian $\mathrm{Kv}$ channels is seldom reported. The four residues, forming the two H-bonds (W434-D447 and T439-Y445) in Shaker channels, are highly conserved among the $\mathrm{Kv}$ channels and may also form $\mathrm{H}$-bonds in mammalian $\mathrm{Kv}$ channels according to the structure of the Kv1.2/Kv2.1 chimeric channel (PDB: 2R9R).

In this study, we tested whether the two H-bonds (W434-D447 and T439-Y445) in the Shaker channels are the key regulators of the slow inactivation for mammalian Kv2.1, Kv2.2, and Kv1.2 channels by point mutation and patch-clamp recording studies.

\section{Materials and Methods}

\subsection{Cell Culture and Transfection}

Human embryonic kidney cells (HEK293) were purchased from the cell bank of the Chinese Academy of Sciences (Shanghai, China). Cells were cultured in Dulbecco's modified Eagle's medium (GIBICO, Grand Island, NY, USA) supplemented with 10\% fetal bovine serum and $1 \%$ antibiotic-antimycotic solution at $37{ }^{\circ} \mathrm{C}$ with $5 \% \mathrm{CO}_{2}$. Plasmids for rat wild-type and mutant $\mathrm{Kv}$ channels were transiently transfected using jetPRIME reagents (Polyplus transfection) according to the manufacturer's instructions. The cells were used for patching experiments $24 \mathrm{~h}$ after transfection.

\subsection{Molecular Biology}

Wild-type rat Kv1.2 (NM_012970.3), Kv2.1 (NM_013186.1), and Kv2.2 (NM_054000.2) cDNAs were subcloned into pEGFPN1 expression vectors. Site-directed mutagenesis for these $\mathrm{Kv}$ channels was achieved by using the Quick-Change XL Site-directed Mutagenesis kit (Stratagene, La Jolla, CA, USA). All mutations were confirmed by sequencing.

\subsection{Electrophysiology}

Whole-cell currents in HEK293 were recorded using an Axopatch 200B amplifier (Axon Instruments, Union City, CA, USA) operated in voltage-clamp mode and data collected with PCLAMP 10.7 software (Axon Instruments). The bath solution contained (in $\mathrm{mM}$ ): $140 \mathrm{NaCl}, 2.5 \mathrm{KCl}, 2.5 \mathrm{CaCl}_{2}, 10$ glucose, 10 HEPES, and $1 \mathrm{MgCl}_{2}$ (pH adjusted to 7.4 using $\mathrm{NaOH}$ ). The internal solution contained (in $\mathrm{mM}$ ): 135 potassium gluconate, $10 \mathrm{KCl}$, $1 \mathrm{CaCl}_{2}, 1 \mathrm{MgCl}_{2}, 10$ HEPES, $2 \mathrm{Mg}$-ATP, and 10 EGTA (pH adjusted to 7.3 using $\mathrm{KOH}$ ). The pipettes were created from capillary tubing (BRAND, Wertheim, Germany) and had resistances of 4 to $6 \mathrm{M} \Omega$ under these solution conditions. Currents were sampled at $10 \mathrm{kHz}$ and filtered at $2 \mathrm{kHz}$ and corrected online for leak and residual capacitance transients using a P/4 protocol. Data from cells with seal resistance $>1 \mathrm{G} \Omega$ and series resistance $<9 \mathrm{M} \Omega$ were included in this study. Series resistance was compensated to $70 \%$ (10-s lag). All of the recordings were performed at room temperature.

\subsection{Western Blot}

Proteins on the surface of HEK293 cells transfected with the wild-type and mutant Kv channels were biotin-labeled and isolated using a Pierce cell surface protein biotinylation and isolation kit (\#A44390, Thermo Scientific, Rockford, IL, USA) according to the manufacturer's instructions. An aliquot of the cell lysate was kept as the total protein fraction. The protein samples were resolved using 10\% SDS PAGE and transferred to polyvinyldifluoride membranes (Millipore, Burlington, MA, USA) in a transfer buffer (25-mM Tris, 192-mM glycine, and $20 \%$ methanol, $v / v$ ) at $100 \mathrm{~V}$ for $1 \mathrm{~h}$. The membranes were blocked with $10 \%$ 
nonfat dry milk in TBST for $1 \mathrm{~h}$ at room temperature. The membranes were incubated with the primary antibody (Anti-Kv1.2, 1:1000 (\#75-008, NeuroMab, Davis, CA, USA); Anti-Kv2.1, 1:500 (\#75-014, NeuroMab); Anti-Kv2.2, 1:1000 (\#APC120AN0150, Alomone Labs, Jerusalem, Israel); and anti-beta tubulin, 1:5000 (\#M20005, Abmart, Shanghai, China)) in an Immunoreaction Enhancer Solution for the primary antibody (TOYOBO, Osaka, Japan) overnight at $4{ }^{\circ} \mathrm{C}$. The membranes were washed 3 times for $10 \mathrm{~min}$ in TBST and incubated with a horseradish peroxidase-conjugated secondary antibody $(1: 10,000)$ (Beyotime, Shanghai, China) in TBST for $2 \mathrm{~h}$ at room temperature. The blots were developed using enhanced chemiluminescence reagents and the ChemiDoc XRS+imaging system from Bio-Rad (Hercules, CA, USA).

\subsection{Statistics}

Data analysis was performed with Clampfit 10.2 (Axon Instruments) and Origin 8.0 software (Origin Lab, Northampton, MA, USA). Statistical analysis consisted of unpaired or paired Student's $t$-tests. Data are given as means $\pm \mathrm{SEM}$, and $n$ indicates the number of tested cells or independent tests. $p<0.05$ was considered statistically significant.

\section{Results}

3.1. Mutation of the Residues Equivalent to W434 and T439 in Shaker Channels Did Not Change Slow Inactivation of Kv2.1, Kv2.2, and Kv1.2 Channels

The four residues, forming two H-bonds (W434-D447 and T439-Y445) in Shaker channels, were conserved among voltage-gated potassium channels. The residue numbers for each channel are shown in Figure 1A. We conducted mutations for two of them in the Kv2.1, Kv2.2, and Kv1.2 channels. As shown in Figure 1B, the W365A and T370A mutations (equivalent to W434 and T439 in Shaker) did not change the slow inactivation of the Kv2.1 channel overexpressed in HEK293 cells. $\mathrm{K}^{+}$currents were elicited by $200-\mathrm{ms}$ steps $(-100$ to $+40 \mathrm{mV}$ in $10-\mathrm{mV}$ increments) applied every $10 \mathrm{~s}$ from a holding potential of $-100 \mathrm{mV}$. The slow inactivation of the Kv2.2 channel was not altered by either W373A or T378A mutations (Figure 1C). The W366A and S371A mutations (Kv1.2 numbering) did not alter the slow inactivation of the Kv1.2 channel overexpressed in HEK293 cells (Figure 1D). The steady-state activation curve (G-V) of the Kv2.1, Kv2.2, and Kv1.2 currents were obtained by plotting the normalized conductance $\left(\mathrm{G} / \mathrm{G}_{\max }\right)$ as a function of the command voltage (Figure $1 \mathrm{E})$. The half-activation potentials $\left(\mathrm{V}_{1 / 2}\right)$ of the $\mathrm{Kv}$ channels were obtained by fitting the conductance with the Boltzmann function. The half-activation potentials $\left(\mathrm{V}_{1 / 2}\right)$ of Kv2.1, Kv2.2, and Kv1.2 were not significantly changed by the mutations $\left(\mathrm{V}_{1 / 2}: \mathrm{Kv} 2.1\right.$, $7.8 \pm 2.1 \mathrm{mV}, \mathrm{Kv} 2.1 \mathrm{~W} 365 \mathrm{~A}, 3.8 \pm 7.4 \mathrm{mV}$, and $\mathrm{Kv} 2.1 \mathrm{~T} 370 \mathrm{~A}, 4.4 \pm 3.7 \mathrm{mV}, n=7-11, p>0.05$, wild type (WT) versus mutants; Kv2.2, $5.7 \pm 1.9 \mathrm{mV}, \mathrm{Kv} 2.2 \mathrm{~W} 373 \mathrm{~A}, 1.7 \pm 3.7 \mathrm{mV}$, and $\mathrm{Kv} 2.2 \mathrm{~T} 378 \mathrm{~A}, 2.1 \pm 5.7 \mathrm{mV}, n=8-14, p>0.05$, WT versus mutants; and $\mathrm{Kv} 1.2,3.2 \pm 2.2 \mathrm{mV}$, $\mathrm{Kv1.2W366A,} 9.1 \pm 4.1 \mathrm{mV}$, and Kv1.2S371A, $6.7 \pm 4.9 \mathrm{mV}, n=6-9, p>0.05$, WT versus mutants. Slope of activation: Kv2.1, $13.0 \pm 0.6 \mathrm{mV}, \mathrm{Kv} 2.1 \mathrm{~W} 365 \mathrm{~A}, 16.3 \pm 2.9 \mathrm{mV}$, and $\mathrm{Kv} 2.1 \mathrm{~T} 370 \mathrm{~A}, 17.2 \pm 4.9 \mathrm{mV}, n=7-11, p>0.05$, WT versus mutants; Kv2.2, $12.1 \pm 0.4 \mathrm{mV}$, Kv2.2W373A, $13.6 \pm 0.9 \mathrm{mV}$, and Kv2.2T378A, $11.6 \pm 1.1 \mathrm{mV}, n=8-14, p>0.05$, WT versus mutants; and $\mathrm{Kv1} 1.2,11.5 \pm 0.7 \mathrm{mV}, \mathrm{Kv} 1.2 \mathrm{~W} 366 \mathrm{~A}, 17.5 \pm 3.1 \mathrm{mV}$, and Kv1.2S371A, $18.5 \pm 5.0 \mathrm{mV}, n=6-9, p>0.05, \mathrm{WT}$ versus mutants). The Kv2.1, Kv2.2, and Kv1.2 channels are slow-inactivating; therefore, longer depolarizing pulse (5s) was used to further test the effects of the mutations on the inactivation.

The inactivation time constants for the wild-type (WT) and mutant Kv channels were determined by fitting a 5-s depolarization at $+20 \mathrm{mV}$ with single exponentials. The W365A and T370A mutations (Kv2.1 numbering) did not change the slow inactivation of the Kv2.1, Kv2.2, and Kv1.2 channels overexpressed in HEK293 cells (inactivation of Kv2.1, $5.6 \pm 1.0 \mathrm{~s}, \mathrm{Kv} 2.1 \mathrm{~W} 365 \mathrm{~A}, 6.0 \pm 1.3 \mathrm{~s}$, and Kv2.1T370A, $6.5 \pm 1.7 \mathrm{~s}, n=6$ to $7, p>0.05, \mathrm{WT}$ versus mutants; Kv2.2, $6.4 \pm 1.6 \mathrm{~s}, \mathrm{Kv} 2.2 \mathrm{~W} 373 \mathrm{~A}, 6.6 \pm 2.2 \mathrm{~s}$, and Kv2.2T378A, $5.8 \pm 1.2 \mathrm{~s}$, $n=6-10, p>0.05$, WT versus mutants; and Kv1.2, $5.3 \pm 1.4 \mathrm{~s}, \mathrm{Kv1}$.2W366A, $4.5 \pm 1.0 \mathrm{~s}$, and Kv1.2S371A, $4.8 \pm 2.1 \mathrm{~s}, n=6-9, p>0.05$, WT versus mutants; Figure 2). The data 
above indicated that the two H-bonds (W434-D447 and T439-Y445) in the Shaker channels are not the key regulators of the slow inactivation for the mammalian Kv2.1, Kv2.2, and Kv1.2 channels.

A

Kv2.1 ASF WWA T I TM T T VGYGD I Y P 381

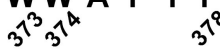

Kv2.2 ASF WWAT I TMT T VGYGD I Y P 389 $3^{60} 3^{0^{0}} \hat{3^{1}}$

Kv1.2 DAF WW A V V SMT T VGYGDMVP 382

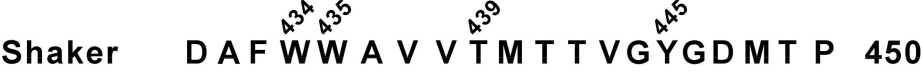

KcsA RAL W $W^{6} W^{6^{8}} S V E \underset{3^{6}}{1^{2}} A T T V G Y 1^{1^{3}} G D L Y P 83$

Kv1.2/2.1 D AF WW A V V SMT T VGYGDMVP 378

B

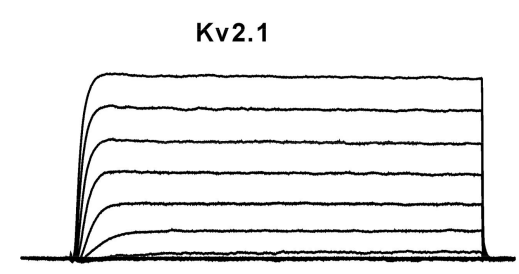

Kv2.1 W365A

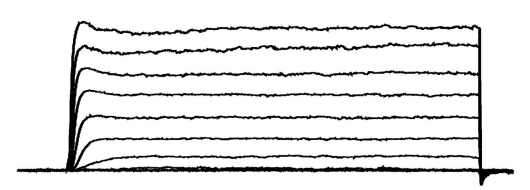

Kv2.1 T370A

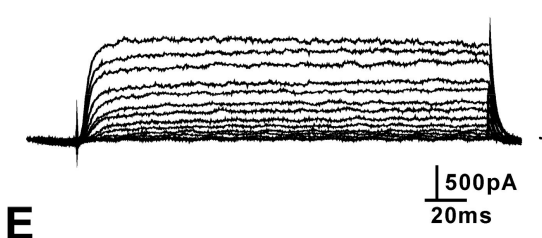

E

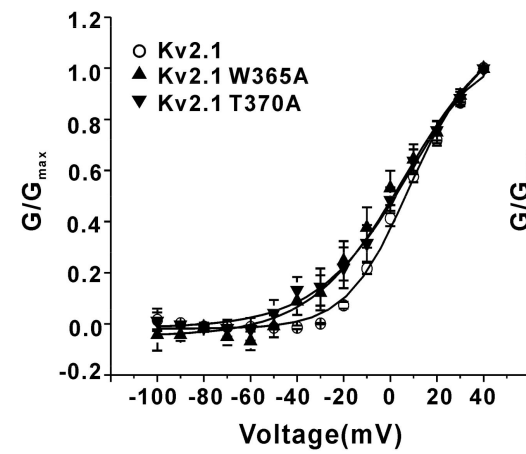

C

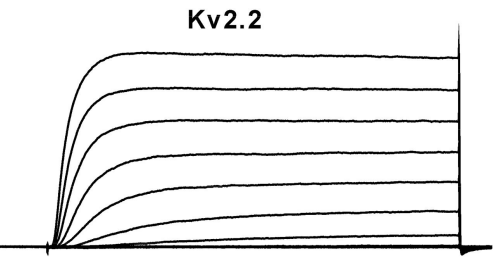

Kv2.2 W373A

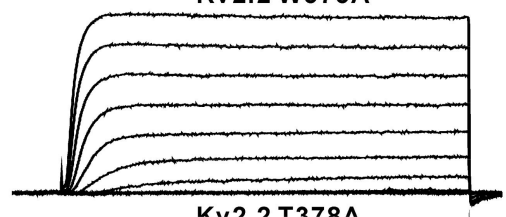

Kv2.2 T378A

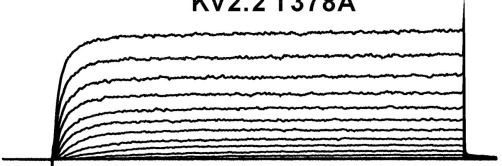

$\frac{1000 \mathrm{pA}}{20 \mathrm{~ms}}$
D

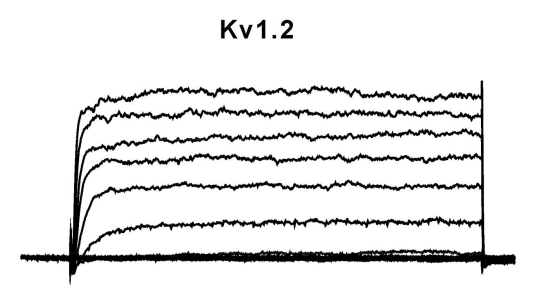

Kv1.2 W366A

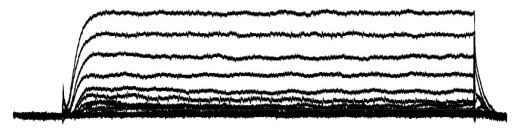

Kv1.2 S371A
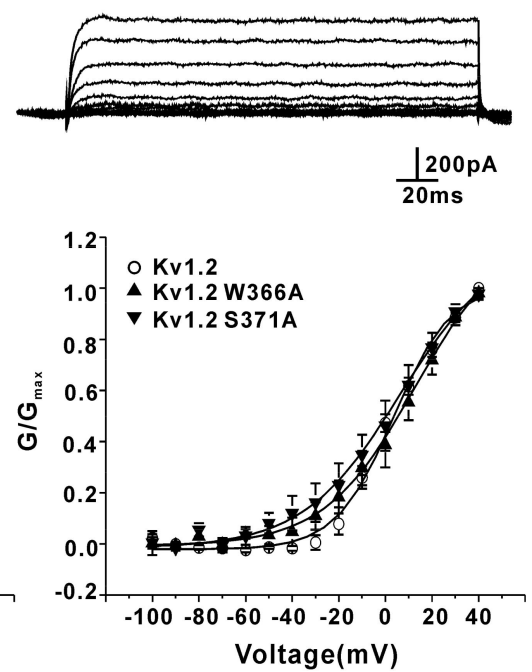

Figure 1. Effects of the mutation of the equivalent residues controlling the slow inactivation of Shaker channels on the rat Kv2.1, Kv2.2, and Kv1.2 channels. (A) Sequence alignment of the selectivity filter and pore helix of various K+ channels: Kv2.1 (GI: 6981120), Kv2.2 (GI: 164663795), Kv1.2 (GI: 25742772), Shaker (GI: 288442), KcsA (GI: 61226909), and the Kv1.2/Kv2.1 chimeric channel (PDB: 2R9R). (B) Representative currents for the Kv2.1 wild-type, W365A, and T370A mutant channels ( -100 to $+40 \mathrm{mV}, 200 \mathrm{~ms}$, in 10-mV increments; $n=8 \sim 14)$. (C) Representative currents for the Kv2.2 wild-type, W373A, and T378A mutant channels $(n=7 \sim 12)$. (D) Representative currents for the Kv1.2 wild-type, W366A, and S371A mutant channels $(n=6-9)$. (E) G-V curves of the wild-type and mutant Kv channels. 
A
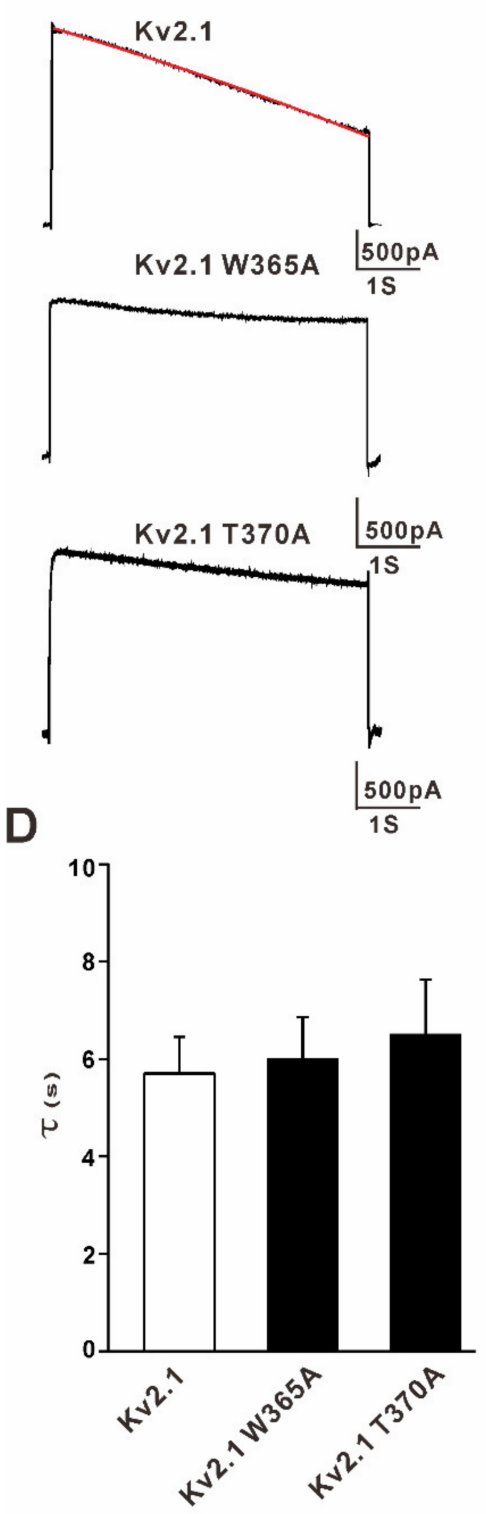

B
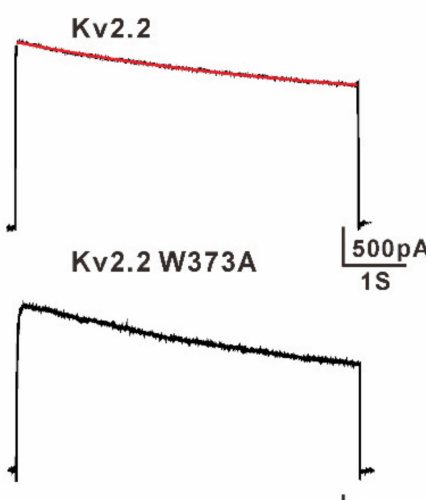

Kv2.2 T378A

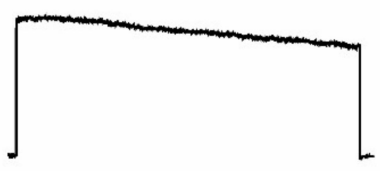

$\frac{400 \mathrm{pA}}{1 \mathrm{~S}}$

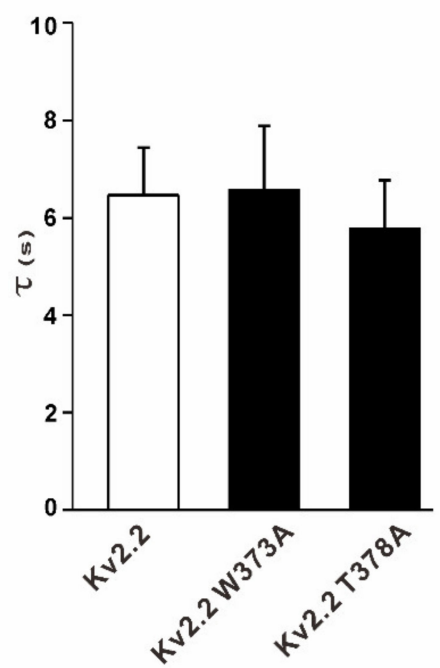

C
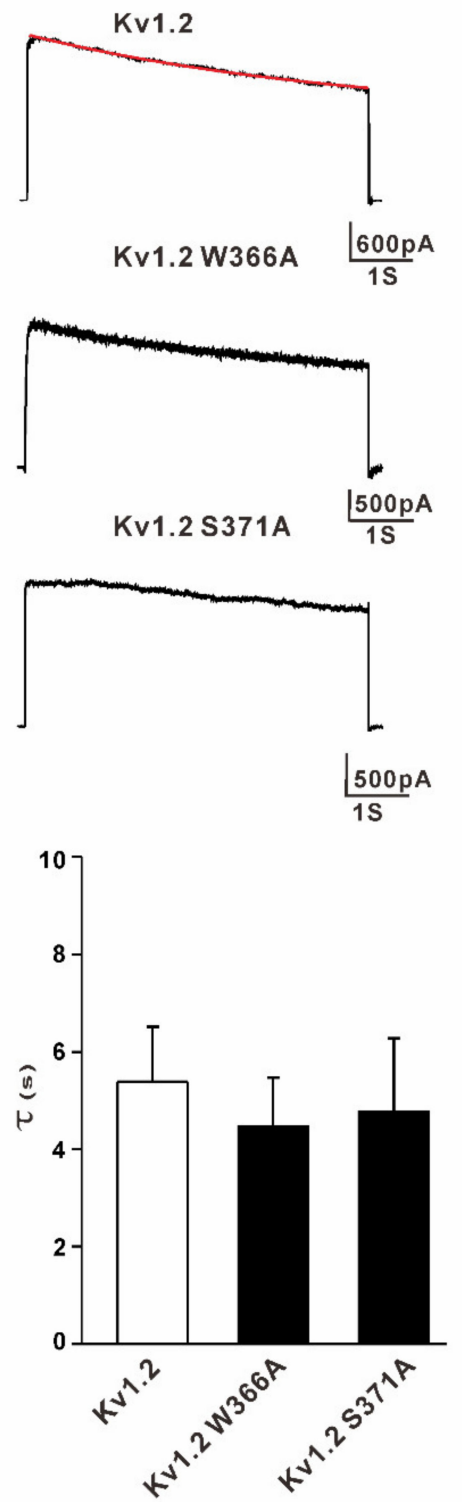

Figure 2. Effects of the mutation of the time constants of the inactivation of the Kv2.1, Kv2.2, and Kv1.2 channels. The inactivation time constants for the wild-type and mutant $\mathrm{Kv}$ channels were determined by fitting a 5-s depolarization at $+20 \mathrm{mV}$ with single exponentials. Red lines show the representative fitting trajectories of the current traces. (A). Representative currents for the Kv2.1 wild-type, W365A, and T370A mutant channels (5-s pulses from - $100 \mathrm{mV}$ to $20 \mathrm{mV}$ ) (B). Representative currents for the Kv2.2 wild-type, W373A, and T378A mutant channels. (C) Representative currents for the Kv1.2 wild-type, W366A, and S371A mutant channels. (D) Comparison of the time constants of the inactivation of the wild-type and mutant Kv channels, $n=6 \sim 12, p>0.05$ (wild type versus mutants).

3.2. Mutation of a Conserved Tryptophan Residue Resulted in the Loss of Function of the Kv2.1, Kv2.2, and Kv1.2 Channels in HEK293 Cells

Structural evidence $[19,20]$ suggests that W366 and Y376 (Kv2.1 numbering) may form an inter-subunit hydrogen bond besides the two H-bonds tested above. The structure of the Kv1.2/Kv2.1 chimeric channel (PDB: 2R9R) shows three putative hydrogen bonds formed by these residues (Figure 3). The Kv1.2/Kv2.1 chimeric channel is derived from a rat Kv1.2 $\mathrm{K}^{+}$channel. It was constructed by replacing the S3b and S4 helices of the Kv1.2 channel with the corresponding voltage-sensor region of the Kv2.1 channel [20]. Therefore, the 
sequence numbering in the chimeric channel was slightly different from that of the Kv1.2 channel. However, the pore region of the chimeric channel was the same as that of the Kv1.2 channel.
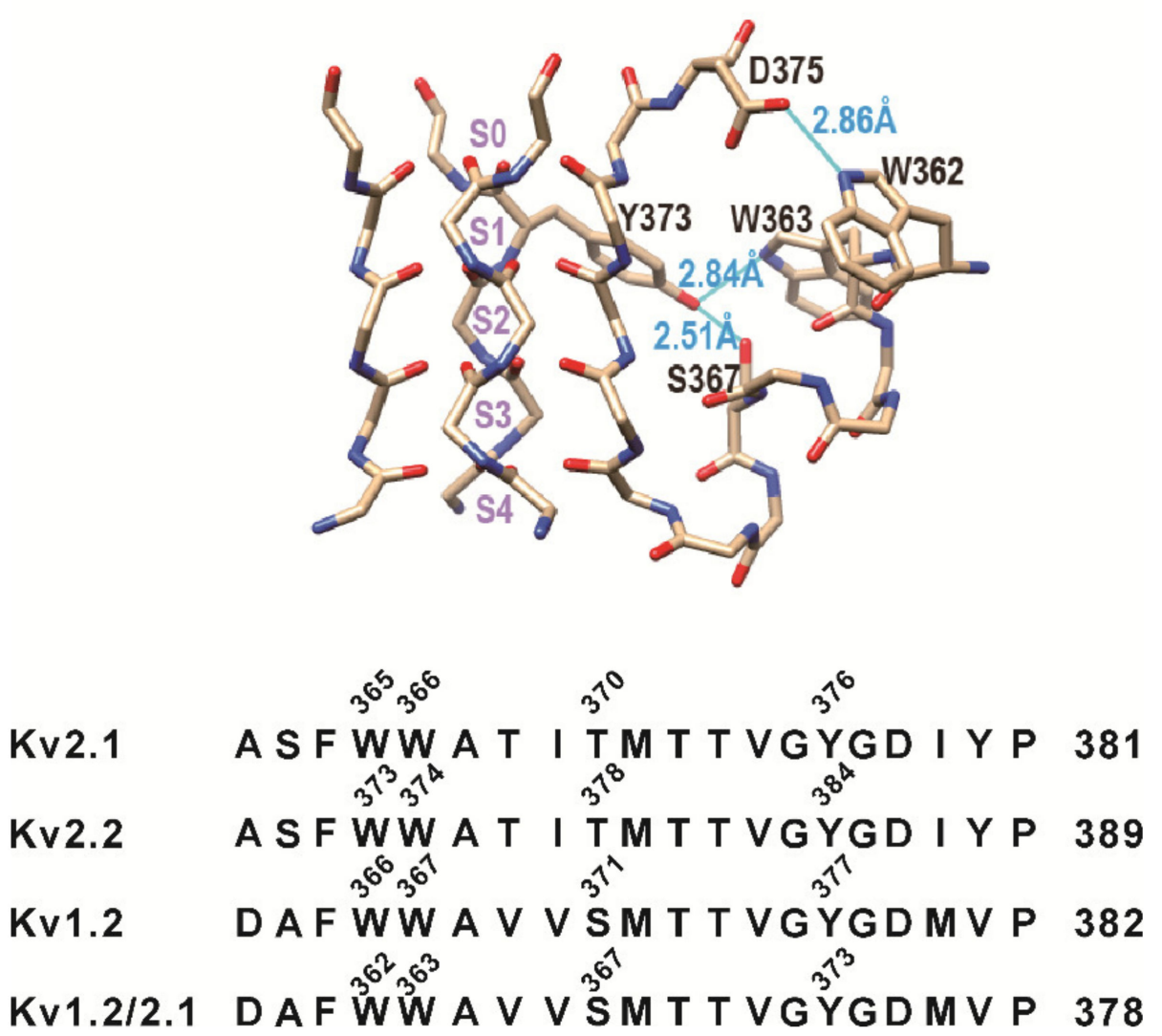

Figure 3. Selectivity filter structure of the Kv1.2/Kv2.1 chimeric channel (PDB: 2R9R) with the ion-binding sites S0-S4 labeled. The relevant pore helix residues and the three putative hydrogen bonds (blue lines) are labeled as well.

The crystal structure shows that W362 can form a hydrogen bond with D375, a residue at the top of the selectivity filter, and W363 and S367 each may form a hydrogen bond with Y373, a residue in the upper portion of the selectivity filter. Thus, they can exert influences on the selectivity filter via these hydrogen bonds. The mutations of these residues may affect the stability, or even the conformation, of the selectivity filter.

We tested the effect of the H-bond (W366-Y376) by the W366A mutation in the Kv2.1 channel. Surprisingly, HEK293 cells transfected with the mutant channels presented no outward potassium currents (Figure 4A), indicating the complete loss of $\mathrm{K}^{+}$conductance or permanent slow inactivation. Next, the W366F mutation was used to test whether the aromatic side chain was involved in this change. As shown in Figure 4A, the W366F mutation had a similar effect on the Kv2.1 channel as W366A did, which suggested that the loss of function might be due to the loss of the H-bond between W366 and Y376. Then, we did similar mutations for the Kv2.2 and Kv1.2 channels. As shown in Figure 4B,C, the W374A/F (Kv2.2) and W367A/F (Kv1.2) mutations produced similar effects. 
A

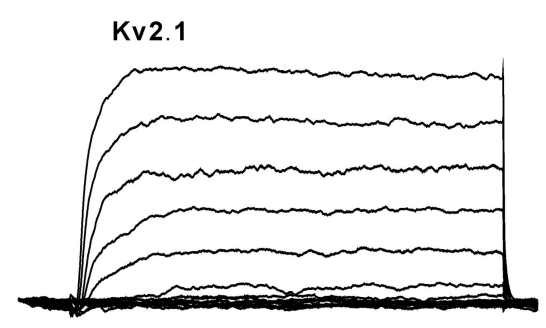

Kv2.1 W366A
B

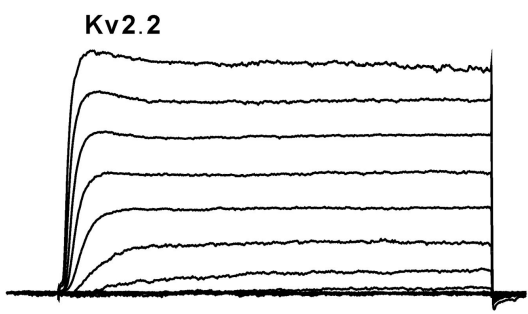

Kv2.2 W374A
C

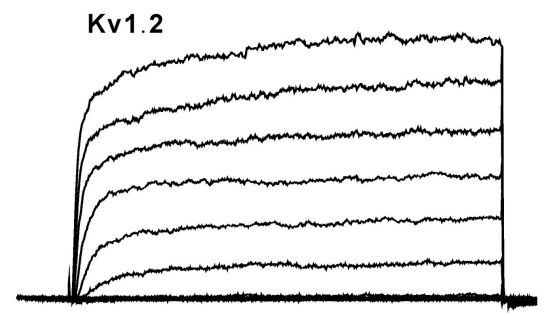

Kv1.2 W367A

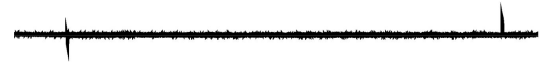

Kv2.1 W366F

Kv2.2 W374F

Kv1.2 W367F
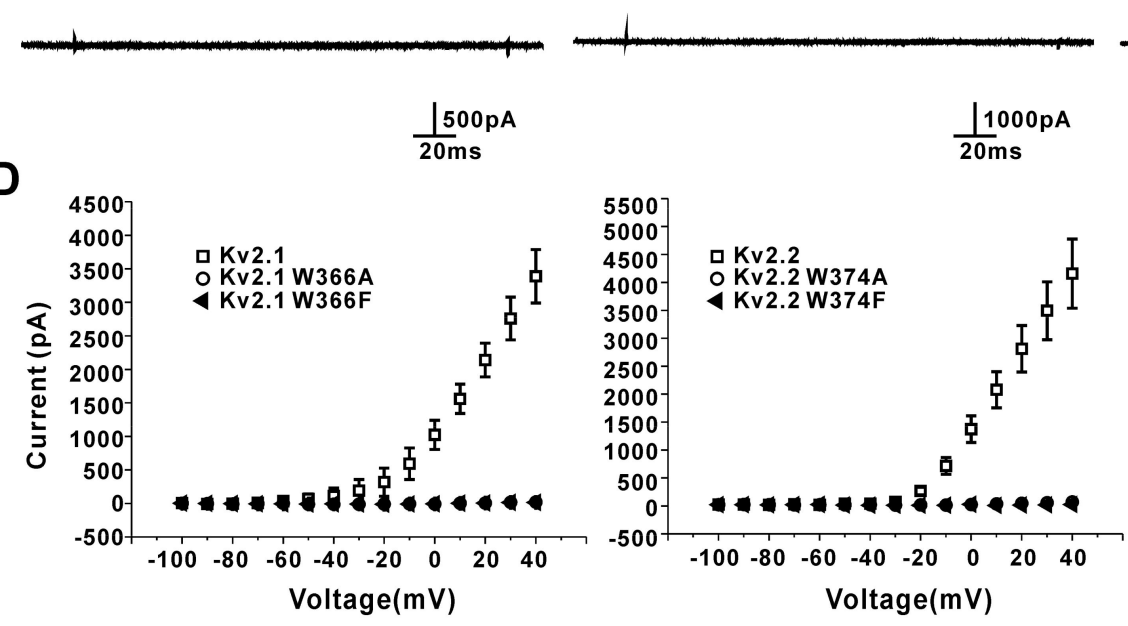

Figure 4. Effects of the mutations of the conserved tryptophan residue on the Kv2.1, Kv2.2, and Kv1.2 channel currents in HEK293 cells. (A) Representative currents for the Kv2.1 wild-type, W366A, and W366F mutant channels. (B) Representative currents for the Kv2.2 wild-type, W374A, and W374F mutant channels. (C) Representative currents for the Kv1.2 wild-type, W367A, and W367F mutant channels. (D) I-V plots of the outward currents of the Kv2.1, Kv2.2, and Kv1.2 wild-type and mutant channels $(n=7-14)$.

The mutant channels showed no obvious outward potassium currents, which might be caused by the point mutation interfering with the protein expression or trafficking to the cell surface. Therefore, we tested the cell surface expression of the various $\mathrm{Kv}$ channels in HEK293 cells. As shown in Figure 5, HEK293 cells do not endogenously express the Kv2.1, Kv2.2, and Kv1.2 channels. In the transfected HEK293 cells, whether with wild-type (WT) or mutant Kv2.1, Kv2.2, and Kv1.2 channels, the surface expression of the Kv channel was clearly detected, and no significant difference was found between the wild-type and mutant channels, indicating that the complete loss of $\mathrm{K}^{+}$conductance shown in Figure 3 was not due to the change of the channel protein expression or the failure of trafficking to the cell surface. 
A

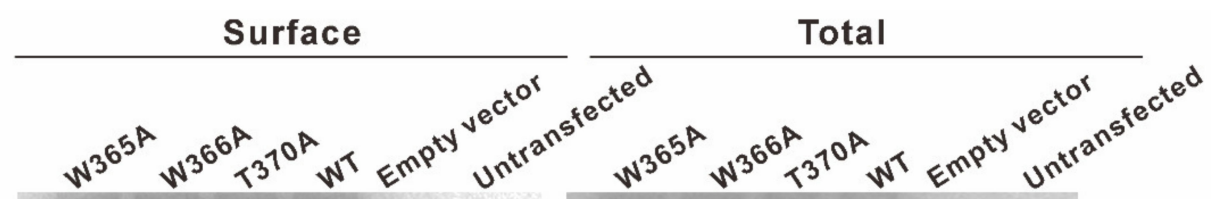

Kv2.1

$n+n^{3} r^{3} \quad h$

$-\infty=0$

Kv2.1

Tubulin

$m-\infty \omega-$ Tubulin

B

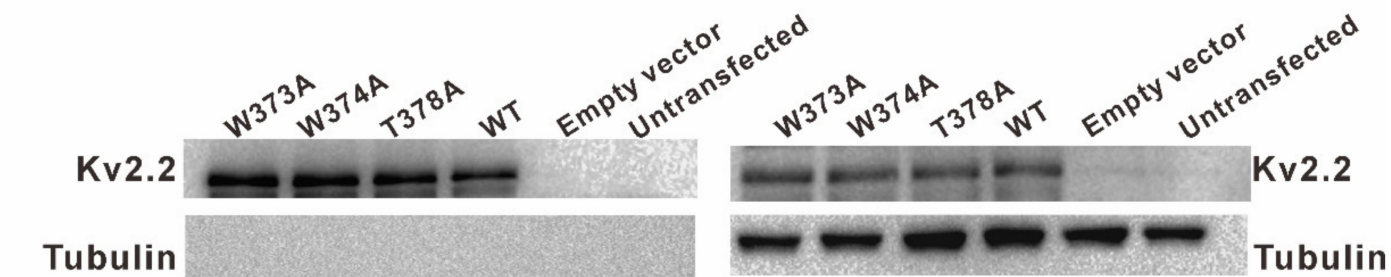

C

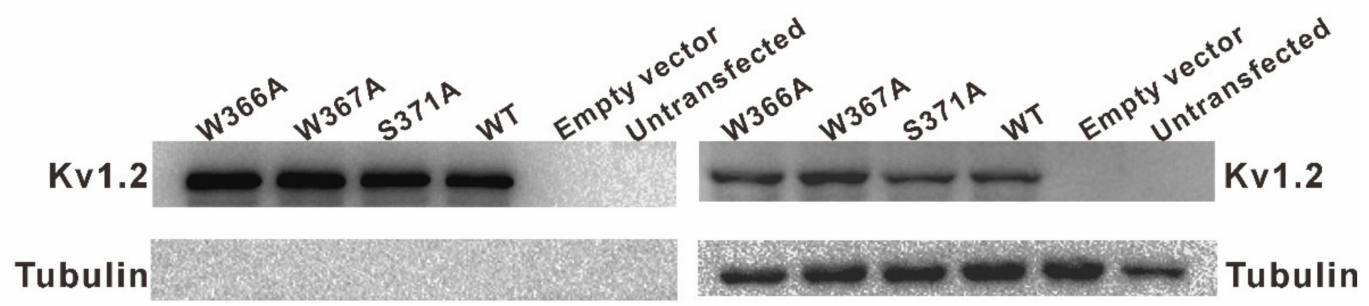

D
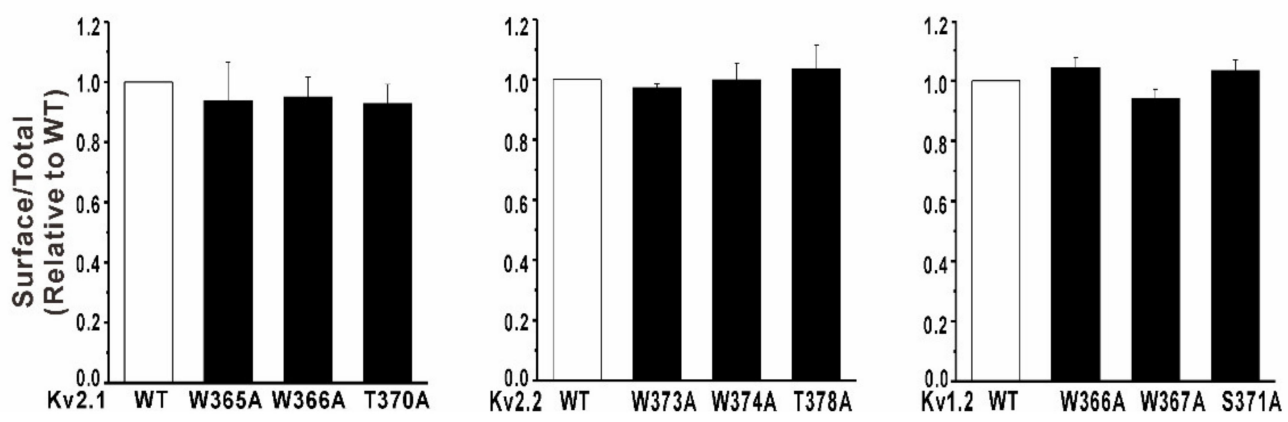

Figure 5. Cell surface expression of the various Kv channels in HEK293 cells. The Kv channel surface expression was detected using the cell surface biotinylation of HEK293 cells transfected with the wild-type/mutant Kv channels. The left panels were the biotinylated membrane protein samples, and the right panels were total protein samples. (A) Sample images showing endogenous or transfected wild-type/mutant Kv2.1 channel expression. (B) Sample images showing endogenous or transfected wild-type/mutant Kv2.2 channel expression. (C) Sample images showing endogenous or transfected wild-type/mutant Kv1.2 channel expression. The signals of the intracellular protein beta-tubulin were detected only in the total protein samples (right panels) and not in the biotinylated surface protein samples (left panels). Endogenous cell surface protein expression of the Kv2.1, Kv2.2, and Kv1.2 channels was undetectable. (D) The mutations did not alter the cell surface expression of the Kv channels in HEK293 cells ( $n=3, p>0.05$, WT versus mutants).

3.3. Y376F (Kv2.1), Y384F (Kv2.2), and Y377F (Kv1.2) Mutations Resulted in Complete Loss of $\mathrm{K}^{+}$Conductance of the Kv Channels Expressed in the HEK293 Cells

If W366 and Y376 form an H-bond that controls the $\mathrm{K}^{+}$conductance of the Kv2.1 channel, the mutation of Y376 should mimic the effect of the W366F mutation. As shown in Figure 6, the Y376F mutant channels indeed showed no potassium current, same as the W366F mutant channels. The Y384F and Y377F mutations resulted in similar effects on the Kv2.2 and Kv1.2 channels, respectively (Figure 6). These data indicate that W366 and Y376 may form an $\mathrm{H}$-bond that controls the $\mathrm{K}^{+}$conductance of mammalian $\mathrm{Kv}$ channels. 
A
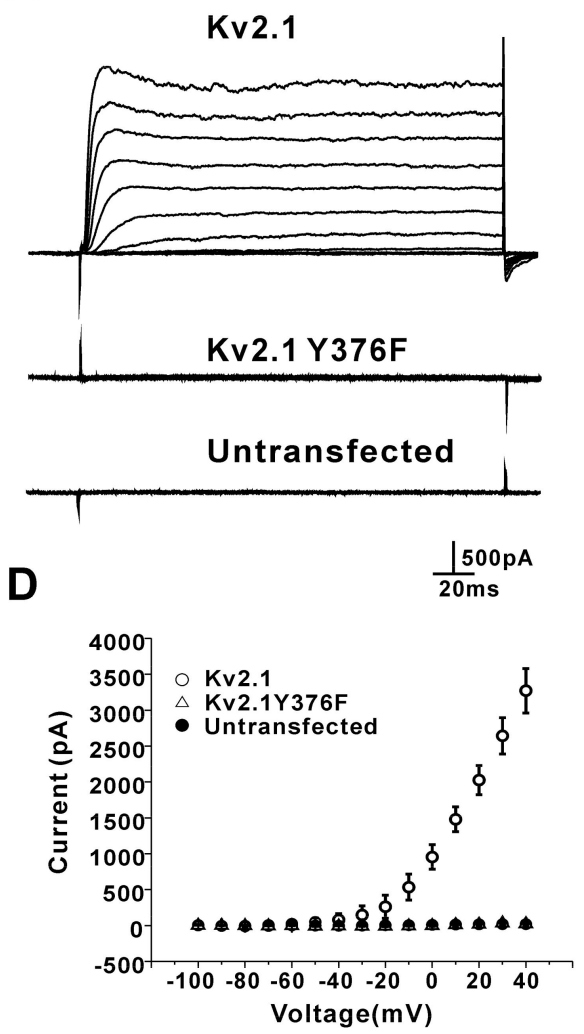

B

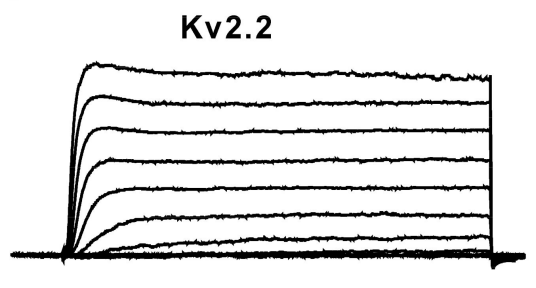

Kv2.2 Y384F
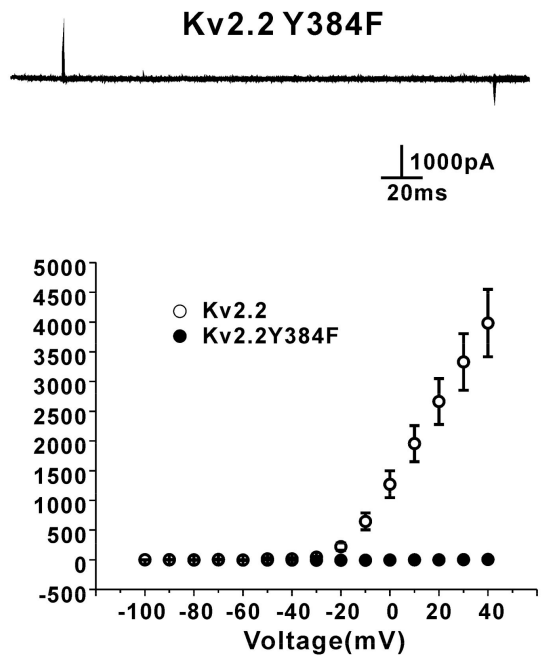

C

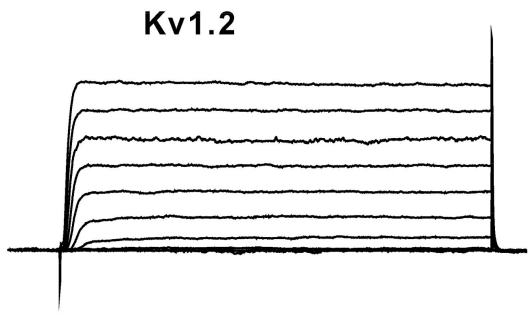

Kv1.2 Y377F
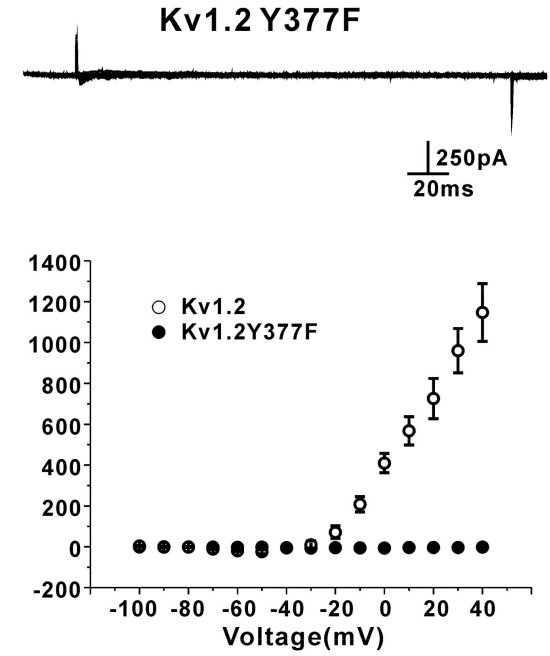

Figure 6. Effects of the mutation of the conserved tyrosine residue on the Kv2.1, Kv2.2, and Kv1.2 channels in HEK293 cells. (A) Y376F (Kv2.1) mutant channels present no obvious outward currents. HEK293 cells present no obvious endogenous outward currents. (B,C) Y384F (Kv2.2) and Y377F (Kv1.2) mutant channels present no obvious outward currents. Currents are elicited by a step protocol (holding at $-100 \mathrm{mV}$ and depolarizing in 10-mV steps from -100 to $+40 \mathrm{mV}$ at 10 -s intervals).

(D) I-V plots of the outward currents of Kv2.1, Kv2.2, and Kv1.2 wild-type and mutant channels $(n=5 \sim 13)$.

\section{Discussion}

In this study, we tested whether the H-bonds controlling the slow inactivation in Shaker channels play important roles in the rat Kv2.1, Kv2.2, and Kv1.2 channels in HEK293 cells. The W365 and D378 residues (Kv2.1 numbering) are highly conserved in Kv channels. The $\mathrm{H}$-bond formed by these two residues plays key roles in controlling the slow inactivation of Shaker channels. However, disrupting the H-bond by point mutation of tryptophan to alanine showed little effect on the slow inactivation in the mammalian Kv2.1, Kv2.2, and Kv1.2 channels expressed in HEK293 cells. Moreover, we checked other electrophysiological properties of these WT and mutant channels; the activation properties of the Kv1.2 and Kv2.1 channels in this study were similar to the previous reported recordings of Kv1.2 and Kv2.1 overexpressed in HEK293 cells [21-23], and, again, no significant differences were found between the WT and the W365A and T370A (Kv2.1) or W366A and S371A (Kv1.2) mutations.

Even though HEK293 cells are a commonly used model system for studying exogenous voltage-gated potassium channels because they have a "null" background, both endogenous voltage-gated $\mathrm{K}+$ channels [24] and Na+ channels [25] in HEK293 cells have been reported previously. The observation of different $\mathrm{Kv}$ expressions in HEK293 may come from different culture conditions or sources of the cells in different labs. We used only a low passage number $(<30)$ of HEK293 cells in the present study. We found very little native $\mathrm{K}^{+}$currents in the HEK293 cells, with occasionally transient tiny sodium currents. Our recordings for the native $\mathrm{K}^{+}$currents in the HEK 293 cells were similar to a previous 
paper [26], which showed that HEK 293 cells with a low passage number $(<45)$ lack endogenous $\mathrm{K}^{+}$channel activity. The cell surface expression of the Kv2.1, Kv2.2, and $\mathrm{Kv} 1.2$ channels was not detected in the nontransfected HEK293 cells, which excluded possible interference by the endogenous $\mathrm{K}^{+}$channels.

Compelling studies have shown that slow inactivation and ionic selectivity are functionally coupled in $\mathrm{Kv}$ channels. The Shaker and Kv2.1 channels displayed decreased $\mathrm{K}^{+}$ and increased $\mathrm{Na}^{+}$permeability during the slow inactivation process $[27,28]$. The W434F mutation in the Shaker channel resulted in the complete loss of $\mathrm{K}^{+}$conductance due to a permanent slow inactivation $[11,12]$ but enabled $\mathrm{Na}^{+}$permeation under an unphysiological condition (in the absence of internal $\mathrm{K}^{+}$) [12,29]. The W435F mutation had no effect on the slow inactivation of Shaker channels [15]. However, in this study, the W366F mutation (equivalent to $\mathrm{W} 435 \mathrm{~F}$ in Shaker channels) in the Kv2.1 channel resulted in no outward $\mathrm{K}^{+}$ currents, indicating a complete loss of $\mathrm{K}^{+}$conductance, which suggests that residues controlling the slow inactivation of mammalian $\mathrm{Kv}$ channels and Shaker channels are different. The Y376F mutation had a similar effect on the Kv2.1 channels as the W366F mutation does. This similar effect occurs in the Kv2.2 and Kv1.2 mutant channels, which indicate that the inter-subunit H-bond formed by W366 and Y376 (by Kv2.1 numbering) plays an important role in the $\mathrm{K}^{+}$permeation of mammalian $\mathrm{Kv}$ channels. Our data did not rule out the possibility that D378 (in Kv2.1 numbering) may play an important role in the slow inactivation of mammalian channels. Besides Y376 and W366 may also form an H-bond with D378, which is worth further investigation.

The selectivity filter is considered as an inactivation gate controlling ion permeation due to its flexibility and its putative conformational changes [5,25,30,31]. In addition, the pore helix residues can also interact with the selectivity filter and influence slow inactivation $[7,11,12,15]$. Based on the crystal structure, the disruption of the W366-Y376 $\mathrm{H}$-bond may lead to an unstable filter or even trigger a conformational change of the selectivity filter. The collapsed filter conformation of the KcsA channel possibly occurs in inactivated channels [8]. Likewise, the W366-Y376 H-bond may influence the filter stability. The absence of potassium currents in the mutant channels may result from the instability of the selectivity filter.

In conclusion, we showed, for the first time, that the inter-subunit $\mathrm{H}$-bond formed by W366 and Y376 (by Kv2.1 numbering) plays an important role in the $\mathrm{K}^{+}$conductance of the mammalian Kv2.1, Kv2.2, and Kv1.2 channels.

Author Contributions: Acquisition and analysis of data, Y.Z., X.Z. and C.L.; conception and design, drafting the article, and supervision, C.H.; and funding acquisition, C.H. All authors have read and agreed to the published version of the manuscript.

Funding: This research was supported by the National Key R\&D Program of China (2016YFA0100802) and the National Natural Science Foundation of China (NSFC 31771282).

Institutional Review Board Statement: Not applicable.

Informed Consent Statement: Not applicable.

Data Availability Statement: All data are contained within this article.

Conflicts of Interest: The authors declare no conflict of interest. The funders had no role in the design of the study; in the collection, analyses, or interpretation of data; in the writing of the manuscript; or in the decision to publish the results.

\section{References}

1. Gutman, G.A.; Chandy, K.G.; Grissmer, S.; Lazdunski, M.; McKinnon, D.; Pardo, L.A.; Robertson, G.A.; Rudy, B.; Sanguinetti, M.C.; Stuhmer, W.; et al. International Union of Pharmacology. LIII. Nomenclature and molecular relationships of voltage-gated potassium channels. Pharmacol. Rev. 2005, 57, 473-508. [CrossRef]

2. Shah, N.H.; Aizenman, E. Voltage-gated potassium channels at the crossroads of neuronal function, ischemic tolerance, and neurodegeneration. Transl. Stroke Res. 2014, 5, 38-58. [CrossRef] [PubMed] 
3. Hayabuchi, Y. The Action of Smooth Muscle Cell Potassium Channels in the Pathology of Pulmonary Arterial Hypertension. Pediatric Cardiol. 2017, 38, 1-14. [CrossRef]

4. Hoshi, T.; Zagotta, W.N.; Aldrich, R.W. Two types of inactivation in Shaker $\mathrm{K}^{+}$channels: Effects of alterations in the carboxyterminal region. Neuron 1991, 7, 547-556. [CrossRef]

5. Liu, Y.; Jurman, M.E.; Yellen, G. Dynamic rearrangement of the outer mouth of a $\mathrm{K}^{+}$channel during gating. Neuron 1996, 16, 859-867. [CrossRef]

6. Loots, E.; Isacoff, E.Y. Protein rearrangements underlying slow inactivation of the Shaker $\mathrm{K}^{+}$channel. J. Gen. Physiol. 1998, 112, 377-389. [CrossRef] [PubMed]

7. Cordero-Morales, J.F.; Jogini, V.; Lewis, A.; Vasquez, V.; Cortes, D.M.; Roux, B.; Perozo, E. Molecular driving forces determining potassium channel slow inactivation. Nat. Struct. Mol. Biol. 2007, 14, 1062-1069. [CrossRef]

8. Cuello, L.G.; Jogini, V.; Cortes, D.M.; Perozo, E. Structural mechanism of C-type inactivation in $\mathrm{K}^{+}$channels. Nature 2010, 466, 203-208. [CrossRef]

9. Cheng, W.W.; McCoy, J.G.; Thompson, A.N.; Nichols, C.G.; Nimigean, C.M. Mechanism for selectivity-inactivation coupling in KcsA potassium channels. Proc. Natl. Acad. Sci. USA 2011, 108, 5272-5277. [CrossRef] [PubMed]

10. Cordero-Morales, J.F.; Jogini, V.; Chakrapani, S.; Perozo, E. A multipoint hydrogen-bond network underlying KcsA C-type inactivation. Biophys. J. 2011, 100, 2387-2393. [CrossRef]

11. Perozo, E.; MacKinnon, R.; Bezanilla, F.; Stefani, E. Gating currents from a nonconducting mutant reveal open-closed conformations in Shaker $\mathrm{K}^{+}$channels. Neuron 1993, 11, 353-358. [CrossRef]

12. Yang, Y.; Yan, Y.; Sigworth, F.J. How does the W434F mutation block current in Shaker potassium channels? J. Gen. Physiol. 1997, 109, 779-789. [CrossRef] [PubMed]

13. Molina, A.; Castellano, A.G.; Lopez-Barneo, J. Pore mutations in Shaker $\mathrm{K}^{+}$channels distinguish between the sites of tetraethylammonium blockade and C-type inactivation. J. Physiol. 1997, 499 Pt 2, 361-367. [CrossRef]

14. Molina, A.; Ortega-Saenz, P.; Lopez-Barneo, J. Pore mutations alter closing and opening kinetics in Shaker $\mathrm{K}^{+}$channels. J. Physiol. 1998, 509 Pt 2, 327-337. [CrossRef]

15. Pless, S.A.; Galpin, J.D.; Niciforovic, A.P.; Kurata, H.T.; Ahern, C.A. Hydrogen bonds as molecular timers for slow inactivation in voltage-gated potassium channels. eLife 2013, 2, e01289. [CrossRef] [PubMed]

16. Lopez-Barneo, J.; Hoshi, T.; Heinemann, S.H.; Aldrich, R.W. Effects of external cations and mutations in the pore region on C-type inactivation of Shaker potassium channels. Recept. Channels 1993, 1, 61-71. [PubMed]

17. Rasmusson, R.L.; Morales, M.J.; Castellino, R.C.; Zhang, Y.; Campbell, D.L.; Strauss, H.C. C-type inactivation controls recovery in a fast inactivating cardiac $\mathrm{K}^{+}$channel (Kv1.4) expressed in Xenopus oocytes. J. Physiol. 1995, 489 Pt 3, 709-721. [CrossRef]

18. Fedida, D.; Maruoka, N.D.; Lin, S. Modulation of slow inactivation in human cardiac Kv1.5 channels by extra- and intracellular permeant cations. J. Physiol. 1999, 515 Pt 2, 315-329. [CrossRef]

19. Doyle, D.A.; Morais Cabral, J.; Pfuetzner, R.A.; Kuo, A.; Gulbis, J.M.; Cohen, S.L.; Chait, B.T.; MacKinnon, R. The structure of the potassium channel: Molecular basis of $\mathrm{K}^{+}$conduction and selectivity. Science 1998, 280, 69-77. [CrossRef] [PubMed]

20. Long, S.B.; Tao, X.; Campbell, E.B.; MacKinnon, R. Atomic structure of a voltage-dependent $\mathrm{K}^{+}$channel in a lipid membrane-like environment. Nature 2007, 450, 376-382. [CrossRef]

21. Al-Sabi, A.; Kaza, S.K.; Dolly, J.O.; Wang, J. Pharmacological characteristics of Kv1.1- and Kv1.2-containing channels are influenced by the stoichiometry and positioning of their $\alpha$ subunits. Biochem. J. 2013, 454, 101-108. [CrossRef]

22. Abraham, M.J.; Fleming, K.L.; Raymond, S.; Wong, A.Y.C.; Bergeron, R. The sigma-1 receptor behaves as an atypical auxiliary subunit to modulate the functional characteristics of Kv1.2 channels expressed in HEK293 cells. Physiol. Rep. 2019, 7, e14147. [CrossRef] [PubMed]

23. Wang, W.; Yin, H.; Feng, N.; Wang, L.; Wang, X. Inhibitory effects of antidepressant fluoxetine on cloned Kv2.1 potassium channel expressed in HEK293 cells. Eur. J. Pharmacol. 2020, 878, 173097. [CrossRef] [PubMed]

24. Jiang, B.; Sun, X.; Cao, K.; Wang, R. Endogenous KV channels in human embryonic kidney (HEK-293) cells. J. Neurosci. Res. 1998, $52,612-617$.

25. He, B.; Soderlund, D.M. Human embryonic kidney (HEK293) cells express endogenous voltage-gated sodium currents and Nav1.7 sodium channels. Neurosci. Lett. 2010, 469, 268. [CrossRef] [PubMed]

26. O'Connella, K.M.S.; Loftusa, R.; Tamkuna, M.M. Localization-dependent activity of the Kv2.1 delayed rectifier $\mathrm{K}^{+}$channel. Proc. Natl. Acad. Sci. USA 2010, 107, 12351-12356. [CrossRef]

27. Starkus, J.G.; Kuschel, L.; Rayner, M.D.; Heinemann, S.H. Ion conduction through C-type inactivated Shaker channels. Mol. Cell. Biochem. 2002, 238, 69-79. [CrossRef]

28. Kiss, L.; LoTurco, J.; Korn, S.J. Contribution of the selectivity filter to inactivation in potassium channels. Biophys. J. 1999, 76, 253-263. [CrossRef]

29. Starkus, J.G.; Kuschel, L.; Rayner, M.D.; Heinemann, S.H. Macroscopic Na ${ }^{+}$currents in the “Nonconducting" Shaker potassium channel mutant W434F. J. Gen. Physiol. 1998, 112, 85-93. [CrossRef] [PubMed]

30. Yellen, G.; Sodickson, D.; Chen, T.Y.; Jurman, M.E. An engineered cysteine in the external mouth of a $\mathrm{K}^{+}$channel allows inactivation to be modulated by metal binding. Biophys. J. 1994, 66, 1068-1075. [CrossRef]

31. Wang, Z.; Wong, N.C.; Cheng, Y.; Kehl, S.J.; Fedida, D. Control of voltage-gated $\mathrm{K}^{+}$channel permeability to NMDG ${ }^{+}$by a residue at the outer pore. J. Gen. Physiol. 2009, 133, 361-374. [CrossRef] [PubMed] 